\title{
Real-Time Cryptocurrency Price Prediction by Exploiting IoT Concept and Beyond: Cloud Computing, Data Parallelism and Deep Learning
}

\author{
Ajith Premarathne ${ }^{1}$, Malka N. Halgamuge ${ }^{2 *}$, R. Samarakody ${ }^{3}$, Ampalavanapillai Nirmalathas $^{4}$ \\ Velrada Capital Pty Ltd, Melbourne VIC $3000^{1}$ \\ Department of Electrical and Electronic Engineering, The University of Melbourne, VIC 3010, Australia ${ }^{2,3,4}$
}

\begin{abstract}
Cryptocurrency has as of late pulled in extensive consideration in the fields of economics, cryptography, and computer science due to it is an encrypted digital currency, peerto- peer virtual forex produced using codes, and it is much the same as another medium of the trade like real cash. This study mainly focuses to combine the Deep Learning with Data parallelism and Cloud Computing Machine learning engine as "hybrid architecture" to predict new Cryptocurrency prices by using historical Cryptocurrency data. The study has exploited 266,776 of Cryptocurrency prices values from the pilot experiment, and Deep Learning algorithm used for the price prediction. The four hybrid architecture models, namely, (i) standalone PC, (ii) Cloud computing without data parallelism (GPU-1), (iii) Cloud computing with data parallelism (GPU-4), and (iv) Cloud computing with data parallelism (GPU-8) introduced and utilized for the analysis. The performance of each model is evaluated using different performance evaluation parameters. Then, the efficiency of each model was compared using different batch sizes. An experimental result reveals that Cloud computing technology exposes new era by performing parallel computing in IoT to reduce computation time up to $90 \%$ of the Deep Learning algorithm-based Cryptocurrencies price prediction model and many other IoT applications such as character recognition, biomedical field, industrial automation, and natural disaster prediction.
\end{abstract}

Keywords-Internet of things; IoT; data parallelism; deep learning; cloud computing

\section{INTRODUCTION}

Cryptocurrency is a technology dominant innovative form of digital currency that secures the financial transactions using cryptography, whereas concealing the identities of its users and minimize the counterfeit of the transactions. Cryptocurrency uses decentralized digital currency control that applies the distributed ledger technology, typically a Blockchain. The blockchain can be a distributed public financial transaction database, a public ledger or digital events that executed and shared between the participating parties. Participants in the Cryptocurrencies market build trust relationships through the formation of Blockchain supported cryptography techniques using hash functions. In 2008, an unknown group or an individual published a paper by introducing themselves under the name of Satoshi Nakamoto and paper entitled Bitcoin: A Peer-To-Peer Electronic Cash System". This paper explains peer-to-peer online electronic cash payment system that would allow sending payments directly from one party to another without involving a financial institution. Bitcoin is the first realized Cryptocurrency concept created in 2009 and thought it extremely popular in 2017 [1]. The price of the Bitcoin has occasionally increased and therefore the value of the bitcoin is considered volatile. Hence, numerous economical entities try to predict the bitcoin price using different tools. This significant price movements of the bitcoin imply the requirement of accurate cryptocurrencies price prediction model to uphold the consistent economic policy. Thus, the demand for the cryptocurrencies price prediction mechanism is high. The cryptocurrencies price prediction model is prevalent around the world because most of the traders in the world use Cryptocurrencies to earn profits in an online market.

The blockchain databases have ready availability a large volume of data however the challenge is analyzing and storing this large volume of data on a time scale. Then, the cloud computing, which is the latest technological evolution of computational science, allowing groups to host, store process, and analyze large volumes of multidisciplinary data. Cloud computing is an internet-based utility service that provides virtualized service, storage, and databases, etc. The cloud technology is a distributed technology platform that leverage to provide highly scalable and resilient environments. Correspondingly, cloud computing architecture supports for the scalability, virtualization, and storage of large volume of structured and unstructured data based on the unlimited resources on demand [2]. Therefore, cloud computing is considered an appropriate platform for deep learning analytics. Google is one of the examples for the major Cloud computing providers. Thus, this study has used the Google Machine Learning (ML) Engine, as a Deep learning computing engine because, the Google ML Engine is easy to instruct for scaled data in deep learning algorithm [3]. Deep Learning referred to as achieved significant scalability and stability and generalization of training on big data. It can develop a model that converts inputs to outputs by extracting complex and nonlinear hierarchical features of training data [4]. The programs of data-parallel entails with a series of operations and functioning to identify the large structured data. However, the parallelism can be either implicit or explicit, and can be regular or irregular [5].

This study aims to combine the Deep Learning with cloud computing and data parallelism based on the IoT concept for the development of cryptocurrencies price prediction model.

*Corresponding Author 


\section{RELATED WORK}

Enormous studies conducted to develop models for the cryptocurrency prices prediction however, there is a considerable gap in the research on predicting cryptocurrency involve with the machine learning algorithms. Many cryptocurrencies price prediction studies $[6,7,8,9]$ performed using standalone computers. However, Geourgoula et al. [10] discussed the Bitcoin price determinants and implemented a sentiment analysis technique that supports vector machines. The author explained that the network hash-rate and the frequency of the Wikipedia views had a significant positive correlation with the fluctuation of the Bitcoin price.

Greaves et al. [11] predicted the Bitcoin price by analyzing the Blockchain using SVM and ANN. The author reported that a regular ANN has 55 percent of price prediction accuracy. The study concluded that exchanges on the outside of the realm of the Blockchain have technically dictated price and it limited the Blockchain data predictability. Similarly, Matta et al. [12] studied the effect of tweets on Twitter and Trend views of Google for the price of Bitcoin with 60 days as sample size and sentiment as a variable. The author found that both Google Trend views and positive tweets have moderately correlated to the Bitcoin price fluctuation and that correlation can be used to predict the cryptocurrencies price. However, the inadequate sample size is a major drawback of the study and prediction based on the social media comments may not be a reliable source for the scientific studies. Steinkrau et al. [13] implemented a GPU-based ANN model and reported that the model is three times faster training and testing than a CPU. Ciresan et al. [14] also reported that GPU-based deep natural network training is forty times faster than a CPU for the image recognition. David Sheehan has proposed a Cryptocurrencies price prediction algorithm [6] based on Long Short-Term Memory (LSTM) neural network model. Correspondingly, Alex (2014) [15] suggested a method for paralleling the training of convolutional neural networks across multiple GPUs.

However, any of these studies did not exploit the IoT basic concepts and cloud computing phenomena in IoT along with Deep Learning for the Cryptocurrencies real-time price prediction. Therefore, this paper explains to quantify the impact of computation time of Deep Learning algorithm training on four models ((i) Standalone PC - (ii) GPU1 without data parallelism model (iii) GPU4 - with data parallelism model and (iv) GPU8 - with data parallelism model) with a high accuracy percentage of Cryptocurrency price prediction. This study mainly focused on Parallel Processing and Cloud computing along with the internet of things (IoT) concept to develop a cryptocurrencies price prediction model.

Main contribution of the paper

- Real-time Cryptocurrency price was predicted by exploiting the Internet of things (IoT) concept and beyond.

- Data parallelism and Cloud Computing Machine learning engine were combined with Deep Learning and this hybrid architecture is applied to
Cryptocurrency historical data to predict new Cryptocurrency price.

- Three hybrid architecture was developed for cryptocurrency data training and predicting purpose (i) standalone PC, (ii) Cloud computing without data parallelism (GUP-1), (iii) Cloud computing with data parallelism (GUP-4).

- Cloud computing technology secure new trends in performing parallel computing in IoT to reduce computation time up to $90 \%$ of the Cryptocurrency price prediction model using Deep Learning algorithm.

- Proposed hybrid architecture can be used in any application including in IoT applications such as character recognition, biomedical field, industry automation and natural disaster prediction.

The rest of the paper is organized as follows: Section II describes related work and the main contribution of the paper. Section III introduces how the data is collected and preprocessed and techniques to combine Deep Learning with cloud computing and data parallelism based on the IoT concept. Section IV provides results, and Section V provides related discussion. Finally, the paper concludes in Section VI.

\section{MATERIALS AND METHODOLOGY}

\section{A. Data Collection and Preparation}

Historical Cryptocurrencies data from the Quandal database collected and recorded daily for four years at different time instances. Then, the data normalized by implementing Min-Max Scalar technique and smoothened over the complete period and normalized data were retrieved up to a current date subsequently. Data preparation performed before the training process by using deep learning algorithm.

Before training the network, the data set scaled to converge the system efficiently. Then, the scaled data set divided into two sets as "training data set" and "testing data set". The deep learning algorithm trained using the training data set and accuracy of the Cryptocurrencies price prediction for an unseen data tested using the testing data set.

The testing data set that manipulated to predict the Cryptocurrencies price trained by creating Neural Network Model which has Five-layers including input, output, and three hidden layers. The ReLU activation function applied for the hidden layers as it can increase the training efficiency. The Liner activation utilized for an output layer as it can pass values without any modification. Then, update the quality and speed of the model parameters using SGD optimizer.

\section{B. Training Methods}

Mean Squared Error (MSE), Mean Absolute Error (MAE), variance and computation time (CPU processing time) computed for each model to identify the best-fitted model to prediction of Cryptocurrencies price.

\section{Performance Evaluation of the Four Models by Comparing the Batch Size}

The MAE values, MSE value, Explained variance Score, Accuracy of the prediction (R2), Min-Max Scalar, Efficiency 
Comparison Percentage and Efficiency of Computation Time of each hybrid architecture model compared using five different size of datasets (batch size) such as (i) Batch 32 (ii) Batch $256 * 4$ (iii) Batch $256 * 8$ (iv) Batch 256*16 and (v) Batch $256 * 32$. The experiment performed for 50, 100, 200, 500, 1000 and 5000 epochs.

\section{Cryptocurrency Price Prediction Mechanism}

Fig. 3 describes the Cloud computing connected Cryptocurrencies predicting mechanism process.

Following steps (Fig. 1) explained the detail procedure for the Cloud computing connected Cryptocurrencies predicting mechanism.

Step 1: Retrieve historical Cryptocurrency data from the internet and save as CSV file.

Step 2: Load historical Cryptocurrency data to the desktop computer.

Step 3: Scale historical Cryptocurrency data to between 01 and then save back as CSV file in the desktop computer.

Step 4-1: Train the Cryptocurrency prediction model using deep learning algorithm without the Cloud computing model.

Step 4-2: Train the Cryptocurrency prediction model using deep learning algorithm in with Cloud computing model or with parallel Cloud computing.

Step 5-1: Save the trained Cryptocurrency prediction model in Cloud computing.

Step 5-2: Save the trained Cryptocurrency prediction model in Cloud computing.

Step 5: Retrieve real-time Cryptocurrency data from the internet as CSV file data.

Step 6: Feed lives Cryptocurrency data to train the Cryptocurrency prediction model that saved in Cloud computing.

Step 7: Get the result back from Cloud computing and show the predicted Cryptocurrency price.

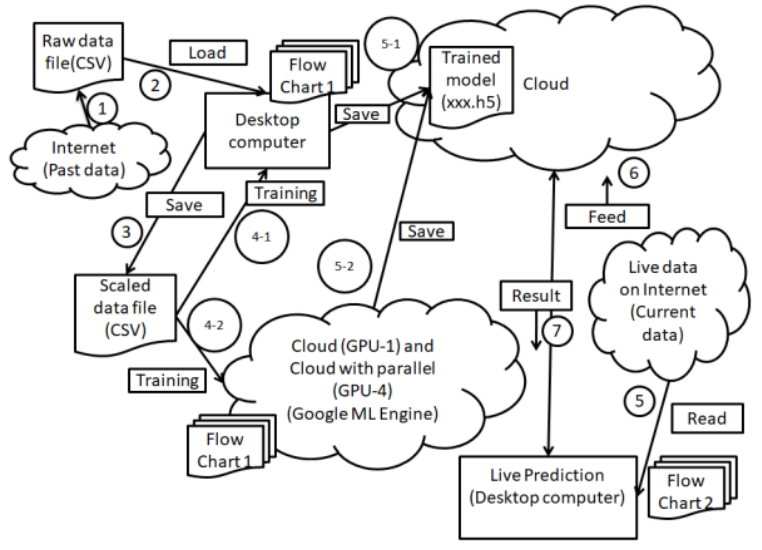

Fig. 1. Cloud Computing Connected Cryptocurrencies Predicting Mechanism using the Deep Learning Algorithm.

\section{E. Deep Learning Training Model in with or without Cloud Computing}

Flow Chart 1 emphasizes in Fig. 5 describes the training phase of the Cryptocurrencies price prediction using deep learning algorithm.

\section{F. Client-Side Cryptocurrency Price Predicting Model}

The Flow Chart 2 shown in Fig. 3 and Fig. 4 describes the prediction algorithm which used for the training method. Then, the training method saved on the Cloud. Finally, this training method used to predict the Cryptocurrencies price for unseen newly arrived data.

\section{G. Data Parallelism Cloud Computing Working Methodology}

The data parallel method explained by [16] has practiced for parallel training as showed in Fig. 2 and steps are as followed.

Step 1: Dataset was divided into eight datasets

Step 2: Feed those data sets into four graphics processing units (GPUs)

Step 3: Each GPU computes different data set of the batches.

Step 4: Data parallelism used synchronization between model parameters and model parallelism doing synchronizing between input and output values between the data chunks.

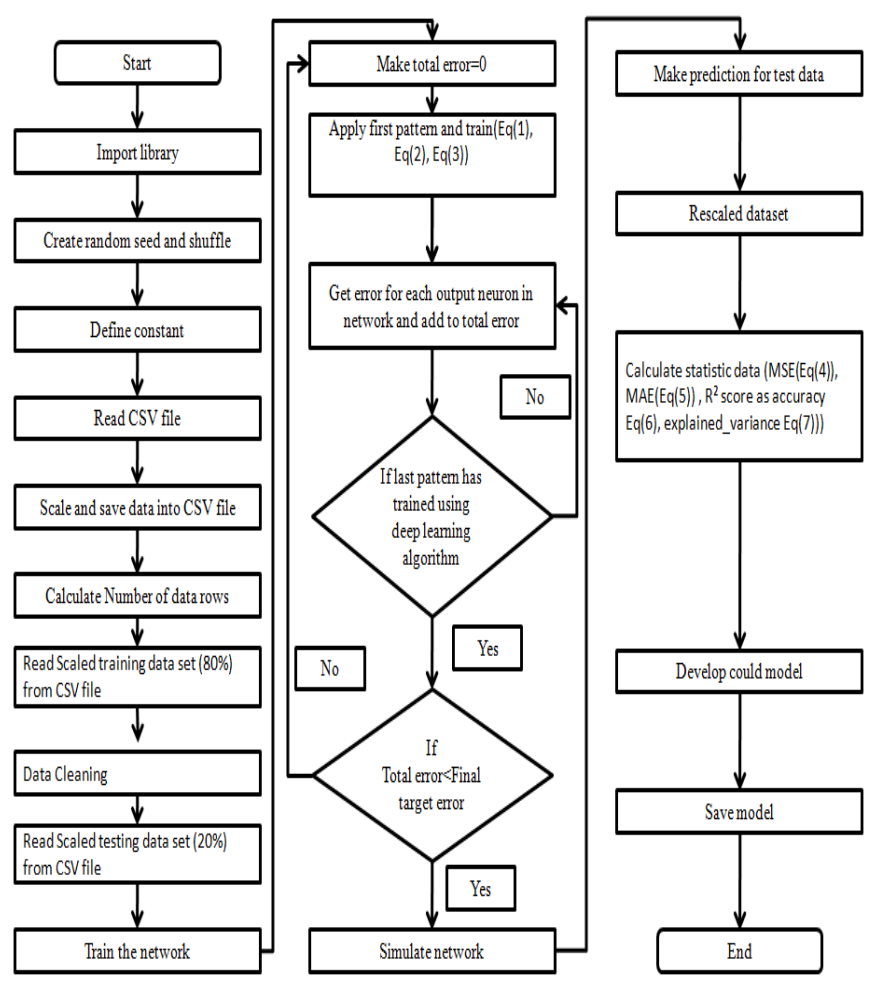

Fig. 2. Server Side: The Cryptocurrencies Price Prediction Training Flow Chart using Deep Learning Algorithm (Flow Chart 1). 


\section{H. Algorithms}

Two algorithms developed for the prediction of Cryptocurrencies price. The algorithm 1 used to compute the MSE, MAE, R2 and explained variance of the historical data and to develop a Cloud computing training model. Then, the training model is developed by the Algorithm 1 (that has saved on the Cloud computing) is used for the Algorithm 2 to predict the Cryptocurrencies price of the live data.

Algorithm 1: Cryptocurrencies price prediction training using Deep Learning algorithm

Begin

Import library

Create random seed and shuffle

Define constant

Read CSV file

Scale and save data into CSV file

Calculate Number of data rows

Read Scaled training data set (80\%) from CSV file

Data Cleaning

Read Scaled testing data set (20\%) from CSV file

Train the network

While total errors $==0$ :

Apply the first pattern and train the network

Get error for each output node in the network and add

to the total error

If the last pattern has trained, then:

If total error < final target error, then:

End training

$$
\text { End If }
$$

End If

\section{End While}

Simulate network

Make a prediction for test data

Rescaled dataset

Calculate statistic data (MSE), MAE, $R^{2}$ score as Cryptocurrency price prediction accuracy, explained variance)

Develop Cloud computing model

Save Cloud computing model

End
Algorithm 2: Cryptocurrencies price predicting using a Deep Learning algorithm

Begin

Import library

Define constant

Initialize the variable

Initialize the plot

Initialize the Google Credentials Variable

While True:

Read live data from the server

Scaled data

For $j$ in range ( 0 , length of the data file): Assign Cloud computing input data Read credential file Gets prediction from Cloud computing Save on data frame

End for

Plot the live prediction graph

Wait for new data

End while

End

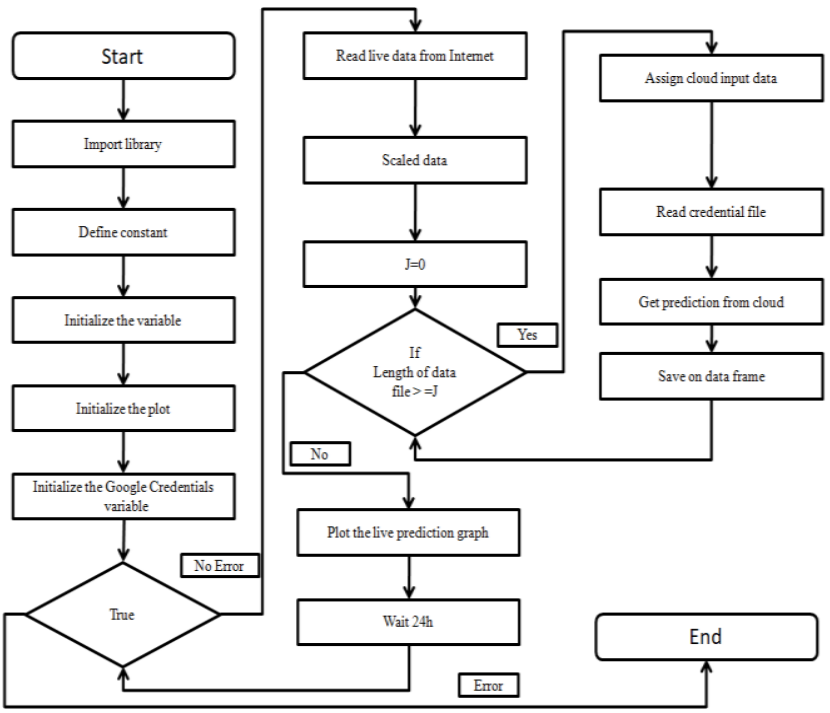

Fig. 3. Client Side: Cryptocurrencies Predicting Flow Chart using Deep.

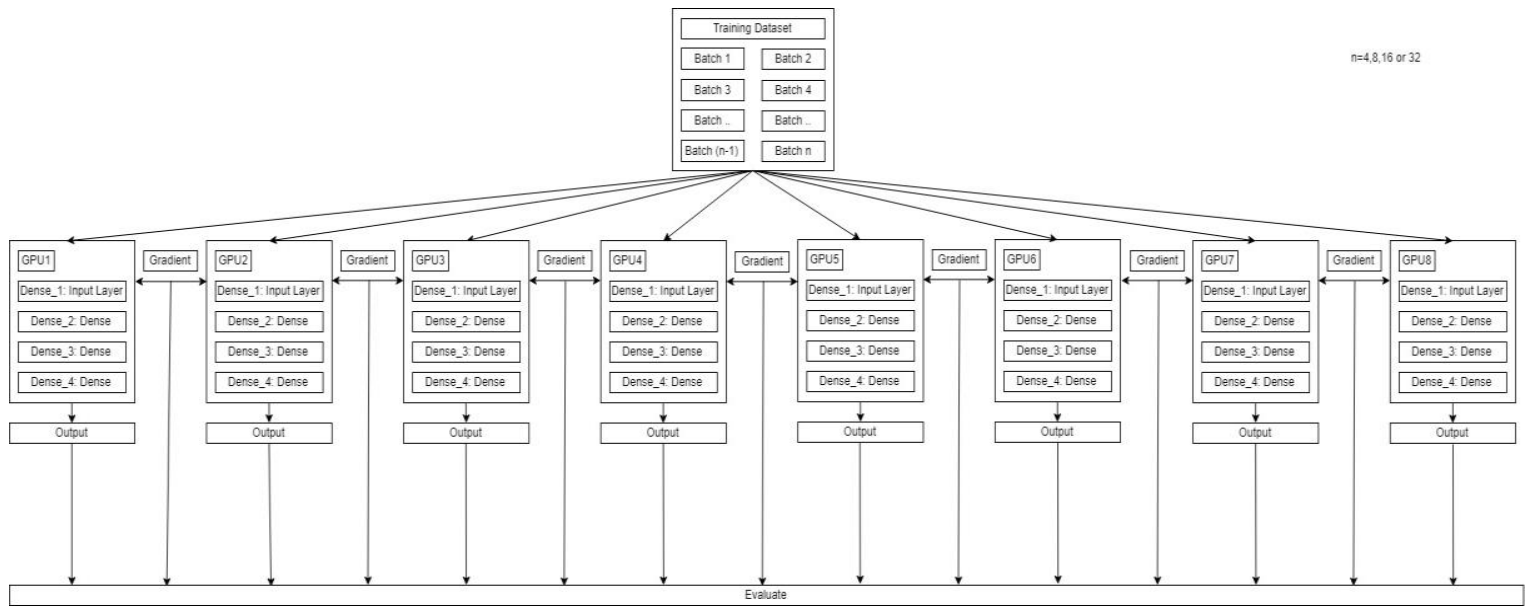

Fig. 4. The Cryptocurrency Data Parallelism Training Block Diagram using Deep Learning Algorithm in Cloud Computing Learning Algorithm (Flow Chart 2). 


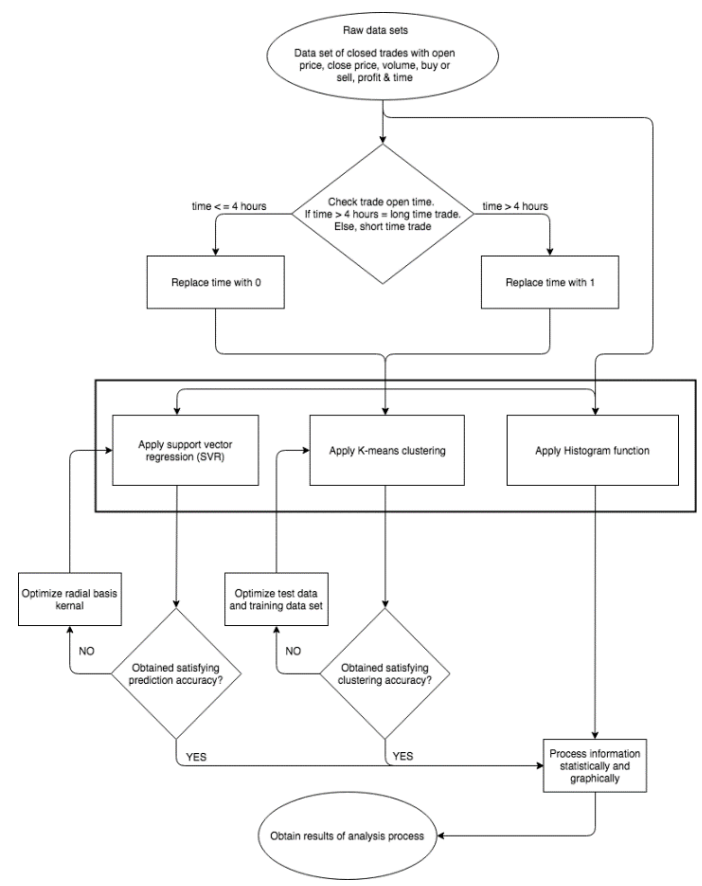

Fig. 5. Overall Methodology (Flow Chart 3).

\section{Comparison of the Efficiency Results}

The efficiency of the model compared using Efficiency Comparison Percentage (ECP) equation and Table I describes the parameters and equation for each step. Fig. 7.

Finally, overall methodology has drafted as showed in

TABLE. I. HYBRID TECHNIQUE: CRYPTOCURRENCY HISTORICAL DATA TRAINING METHODS

\begin{tabular}{|c|c|c|c|c|}
\hline Method & \multicolumn{3}{|l|}{ CPU } & $\begin{array}{l}\text { Memory } \\
\text { (GB) }\end{array}$ \\
\hline Standalone PC & \multicolumn{3}{|c|}{ Intel core i3 - $7100 \mathrm{U}-2.4 \mathrm{~Hz}$} & 8 \\
\hline $\begin{array}{l}\text { Cloud computing } \\
\text { Method }\end{array}$ & GPU name & $\begin{array}{l}\text { GPU } \\
\text { model }\end{array}$ & GPUs & $\begin{array}{l}\text { GPU } \\
\text { memory } \\
\text { (GB) } \\
\text { (GDDR5) }\end{array}$ \\
\hline $\begin{array}{l}\text { Cloud computing } \\
\text { without data } \\
\text { parallelism } \\
\text { (GPU1 - without } \\
\text { data parallelism } \\
\text { model) }\end{array}$ & $\begin{array}{l}\text { Optimizer SGD } \\
\text { (Stochastic } \\
\text { gradient descent): } \\
\text { In each training, } \\
\text { SGD will update } \\
\text { the parameter } \\
\text { Standard_GPU }\end{array}$ & $\begin{array}{l}\text { NVIDI } \\
\text { A } \\
\text { Tesla } \\
\text { K80 }\end{array}$ & 1 & 12 \\
\hline $\begin{array}{l}\text { Cloud computing } \\
\text { with data } \\
\text { parallelism } \\
\text { (GPU4) } \\
\text { (GPU4 - with } \\
\text { data parallelism } \\
\text { model) }\end{array}$ & $\begin{array}{l}\text { Complex_model_1 } \\
\text { _GPU }\end{array}$ & $\begin{array}{l}\text { NVIDI } \\
\text { A } \\
\text { Tesla } \\
\text { K80 }\end{array}$ & 4 & 48 \\
\hline $\begin{array}{l}\text { Cloud computing } \\
\text { with data } \\
\text { parallelism } \\
\text { (GPU8) } \\
\text { (GPU8 - with } \\
\text { data parallelism } \\
\text { model) }\end{array}$ & $\begin{array}{l}\text { Complex_model_1 } \\
\text { _GPU }\end{array}$ & $\begin{array}{l}\text { NVIDI } \\
\text { A } \\
\text { Tesla } \\
\text { K80 }\end{array}$ & 8 & 120 \\
\hline
\end{tabular}

\section{RESULT}

\section{A. Performance Evaluation of the Four Models by Comparing the Batch Size}

1) Comparing the performance evaluation of the standalone PC method: According to Fig. 6 the batch 32 recorded 88.706 of the highest prediction accuracy value in epoch 5000 and it consumes 765.690 minutes while the batch $256 * 32$ recorded 35.886 as the lowest accuracy rate in epoch 50 during 41.552 minutes. According to the results of these comparisons, the highest prediction accuracy value observed from Batch $256 * 4$ as 85.646 while it consumed 81.030 minutes in epoch 5000. However, Batch 256*16 has significant prediction accuracy of 81.266 and efficiency is 59.78 minutes.

2) Comparing the performance evaluation of the GPU1 without data parallelism: Fig. 7 indicates that the maximum and minimum prediction accuracy values of the GPU1 without data parallelism models observed in epoch 5000 and 50 respectively for all batch sizes. However, batch 32 recorded, 88.703 as maximum prediction accuracy value and consume 1785.97 minutes. The batch $256 * 32$ had 35.874 as minimum prediction accuracy value and it used 7.624 minutes. Conferring to the results in Fig. 7, epoch 5000 reported 85.647 of prediction accuracy as the highest value in Batch $256^{*} 4$ while it consumes 152.983 minutes to fulfill the target efficiency. However, for the GPU1 - without data parallelism model the Batch $256 * 16$ reached 81.267 accuracy percentage.

3) Comparing the performance evaluation of the GPU4 with data parallelism: Fig. 8 emphasizes the accuracy value comparison of the five batches. According to the result, the Batch 32 has 87.779 of the highest prediction accuracies in epoch 5000 while the Batch $256 * 32$ has 35.874 of prediction accuracy which is reported as the lowest.

4) Comparing the performance evaluation of the GPU8 with data parallelism: The highest prediction accuracy of 87.071 reported by the batch 32 in epoch 5000 and consumed 686.541 minutes (Fig. 9). However, the batch $256 * 32$ has the best efficiency which is 28.23 minutes and prediction accuracy of 79.088 for the GPU8 - with data parallelism model.

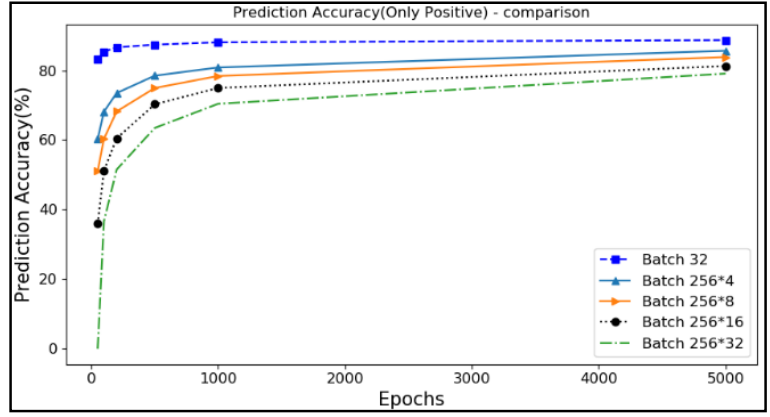

Fig. 6. Comparing the Prediction Accuracy Values of the Standalone PC Method Related to different batch Size. 


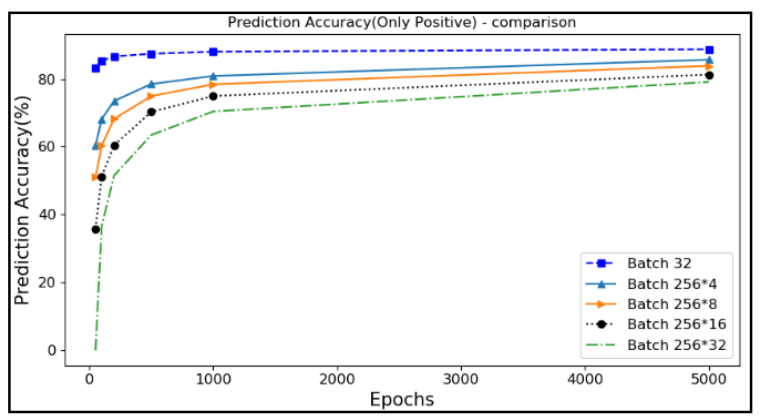

Fig. 7. Comparison of the Prediction Accuracy of the GPU1 - without Data Parallelism whereas; Parallelism - Efficiency (without Batch 32) Values Related to different Batch Size.

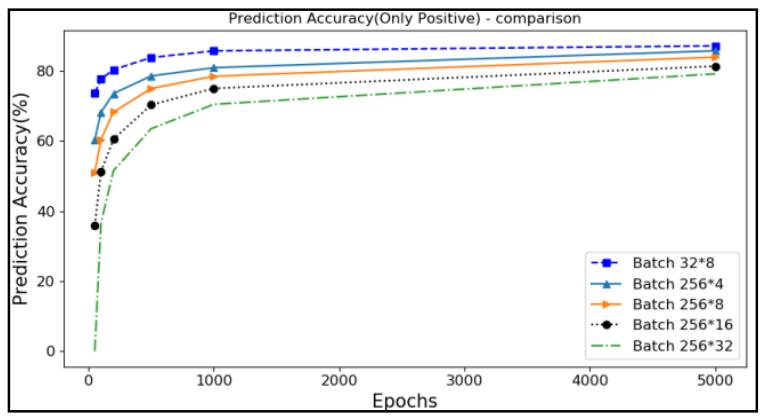

Fig. 8. Comparison of the - Prediction Accuracy Values of the GPU4 - with Data Parallelism Related to different Batch Size.

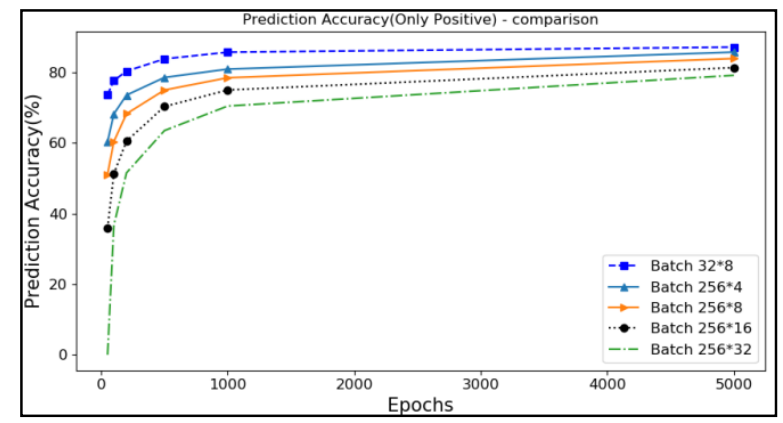

Fig. 9. Comparison of the Prediction Accuracy Values of the GPU8 - with Data Parallelism Related to different Batch Size.

\section{B. Comparison of the Efficiency Percentage Results}

Efficiency percentage of each model was compared using the methodology described in the Table II.

Method A; Cloud computing without data parallelism (GPU1) model vs. Cloud computing with data parallelism model (GPU4).

Method B; Cloud computing without data parallelism (GPU1) model vs. Standalone PC model.

Method C; Cloud computing without data parallelism (GPU1) model vs. Cloud computing with data parallelism model (GPU8).

Method D; Standalone PC model vs. Cloud computing with data parallelism model (GPU4).

Method E; Standalone PC model vs. Cloud computing with data parallelism model (GPU8).
Method F; Cloud computing with data parallelism (GPU4) model vs. Cloud computing with data parallelism model (GPU8).

1) Efficiency comparison of the algorithms for batch 256*8: Fig. 10 reveals that the EPC results from Method A to Method $\mathrm{F}$ for the Batch $256 * 8$. The GPU1 has no data parallelism, therefore, it spent a lot of time on the training compared to the GPU4. However, the ratio of Method A is significantly higher than Method B, Method C, Method D, and Method F. In Method B, the GPU1 model runs in cloud platform and the Standalone PC without cloud just like a laptop computer. The GPU1 module consumed more time for the training compared with Standalone PC model because the GPU1 module requires considerable time to flush the memory. The Standalone PC model has higher efficiency percentage from 50 to 200 epochs while runs faster within that epochs range than the GPU4 model. Subsequently, the efficiency percentage of the Standalone PC model slightly slower than GPU4 model. Hence, until 200 epochs Method B ratio is higher than Method A. The GPU1 model in Method C took more time for the training the GPU8 model because it does not include the data parallelism. However, GPU8 model is slightly slower than the GPU4 and Standalone PC models; thus, the ratio of the Method C comparatively lower than Method A, Method B, and Method F.

TABLE. II. COMPARISON EQUATIONS FOR EFFICIENCY COMPARISON PERCENTAGE (ECP)

\begin{tabular}{|c|c|c|}
\hline $\begin{array}{l}\text { Comparison } \\
\text { Method }\end{array}$ & Description & $\begin{array}{l}\text { The equation for Efficiency } \\
\text { Comparison Percentage } \\
\text { (ECP) calculation }\end{array}$ \\
\hline Method A & $\begin{array}{l}\text { Cloud computing without } \\
\text { data parallelism (GPU1) } \\
\text { model vs. Cloud } \\
\text { computing with data } \\
\text { parallelism model } \\
\text { (GPU4) }\end{array}$ & $\begin{array}{l}\text { Percentage }=[\text { (Cloud } \\
\text { computing without data } \\
\text { parallelism (GPU1)- Cloud } \\
\text { computing with data parallelism } \\
(\text { GPU4)) /Cloud computing } \\
(\text { GPU1) } * 100 \%\end{array}$ \\
\hline Method B & $\begin{array}{l}\text { Cloud computing without } \\
\text { data parallelism (GPU1) } \\
\text { model vs. Standalone PC } \\
\text { model }\end{array}$ & $\begin{array}{l}\text { Percentage }=[\text { (Cloud } \\
\text { computing (GPU1) - standalone } \\
\text { PC) } / \text { Cloud computing (GPU } \\
1)] * 100 \%\end{array}$ \\
\hline Method C & $\begin{array}{l}\text { Cloud computing without } \\
\text { data parallelism (GPU1) } \\
\text { model vs. Cloud } \\
\text { computing with data } \\
\text { parallelism model } \\
\text { (GPU8) }\end{array}$ & $\begin{array}{l}\text { Percentage }=[\text { (Cloud } \\
\text { computing without data } \\
\text { parallelism (GPU1)- Cloud } \\
\text { computing with data parallelism } \\
(\text { GPU8) } / \text { Cloud computing } \\
(\text { GPU1) } * 100 \%\end{array}$ \\
\hline Method D & $\begin{array}{l}\text { Standalone PC model vs. } \\
\text { Cloud computing with } \\
\text { data parallelism model } \\
\text { (GPU4) }\end{array}$ & $\begin{array}{l}\text { Percentage }=[(\text { Standalone PC - } \\
\text { Cloud computing with data } \\
\text { parallelism }(\mathrm{GPU} 4)) / \\
\text { Standalone PC }] * 100 \%\end{array}$ \\
\hline Method E & $\begin{array}{l}\text { Standalone PC model vs. } \\
\text { Cloud computing with } \\
\text { data parallelism model } \\
\text { (GPU8) }\end{array}$ & $\begin{array}{l}\text { Percentage }=[(\text { Standalone PC - } \\
\text { Cloud computing with data } \\
\text { parallelism }(\mathrm{GPU} 8)) / \\
\text { Standalone PC }] * 100 \%\end{array}$ \\
\hline Method F & $\begin{array}{l}\text { Cloud computing with } \\
\text { data parallelism (GPU4) } \\
\text { model vs. Cloud } \\
\text { computing with data } \\
\text { parallelism model } \\
\text { (GPU8) }\end{array}$ & $\begin{array}{l}\text { Percentage }=[\text { (Cloud } \\
\text { computing with data parallelism } \\
(\text { GPU8)- Cloud computing with } \\
\text { data parallelism (GPU4)) } \\
\text { /Cloud computing (GPU8) }{ }^{*} \\
100 \%\end{array}$ \\
\hline
\end{tabular}


Efficiency Percentage comparison results of Method D indicated that the Standalone PC model required more time for the training compared to GPU4 model. In Method D, the efficiency of both GPU4 and Standalone PC models faster than the GPU1 and GPU8 models. Therefore, Method D ratio is the lowest due to comparing the two fastest algorithms. According to the equation of Method E, Standalone PC model is faster than GPU8 model; hence, the Method E ratio is negative and Method E line is not plot in Fig. 10. The GPU8 model in Method consumed considerable time for the training compared to the GPU4 model. The inter-process communication of the GPU8 model may be the reason for this substantial time consumption and as a result Method F ratio is well above Method C and Method D.

2) Efficiency comparison of the algorithms in batch 256*16: The GPU1 model in Method A has no data parallelism therefore it spent lot time for the training than the GPU4 model and the efficiency percentage of the GPU1 model in Batch $256 * 16$ is significantly slower than the Batch $256 * 8$ (Fig. 11). However, the ratio of Method A is higher than the other methods. As in Batch $256 * 8$ for Method B, the GPU1 model spent more time on the training than the Standalone PC model because the GPU1 model consumed considerable time to flush the memory. Results of the Method B in Batch 256*16 has evidently shown that the Standard PC model is slower than the GPU4 and GPU8 model and therefore, the ration of the Method B tracked below the Method A and Method C. In Method C, the GPU8 model is slightly speed than the Standalone PC model, however, the efficiency percentage of the GPU1 model in Method C for the Batch 256*16 showed comparatively higher efficiency percentage than the Batch $256 * 8$. Thus, the Method C ratio is slightly below than the Method.

Method D result illustrated that the Standalone PC model is $50 \%$ slower than the GPU4 model. Therefore, Method D ratio is lower than Method A, Method C, and Method B while higher than the Method E and Method F. The efficiency ratio of the Standalone PC model in Method E is slightly slower than the GPU8 model hence, Method E ratio is above Method F. The GPU8 model in Method F consumed substantial time for the training than the GPU4 model. It caused to slower the GPU8 model and inter-process communication may be the reason for this significant time consumption. As a result, the Method F ratio is lowest for the Batch 256*16.

3) Efficiency percentage comparison of the algorithms in batch 256*32: In Method A, GPU1 spent significant time on the training compared to the GPU4 model (Fig. 12). However, the ratio of Method A is significantly higher than other methods. The GPU1 model in Method B runs in cloud platform and it consumed more time for the training compared with the Standalone PC model because the GPU1 model take some time to flush the memory. The efficiency ratio of the GPU8 model in Method $\mathrm{C}$ is slightly speeding than the Standalone PC model, therefore, the ratio of the Method C is lower than the Method A and higher than the Method B, Method D, Method E, and Method F.

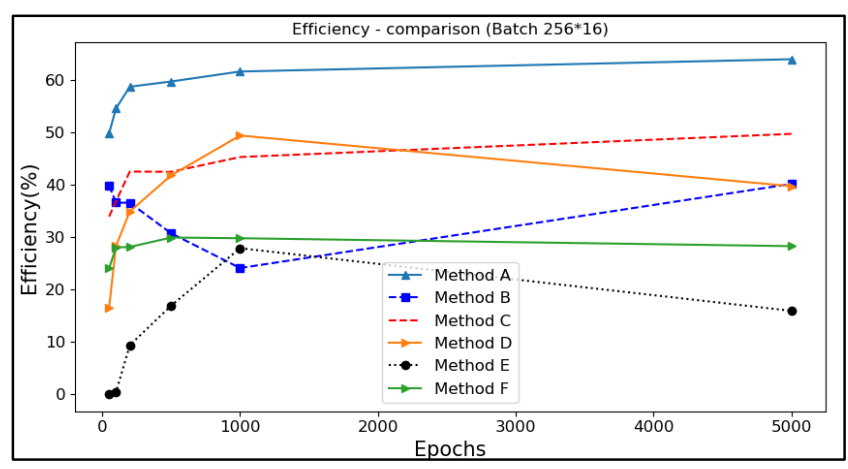

Fig. 10. Efficiency Comparison Percentage for the Batch $256 * 8$.

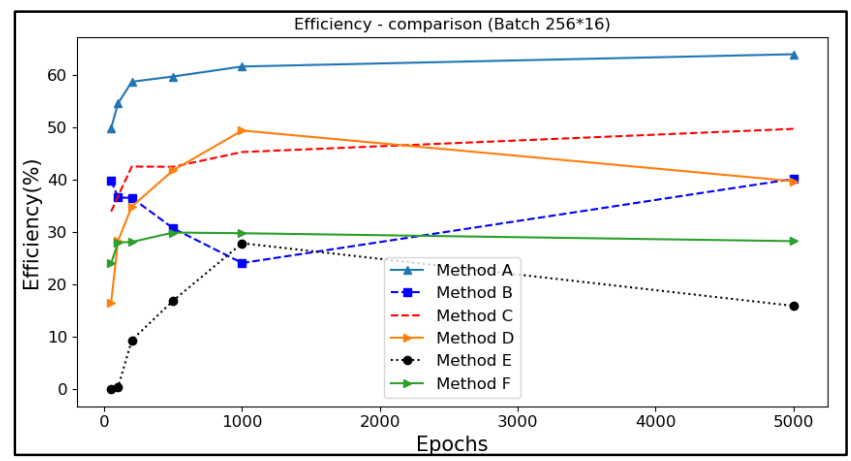

Fig. 11. Efficiency Percentage Comparison for the Batch $256^{*} 16$.

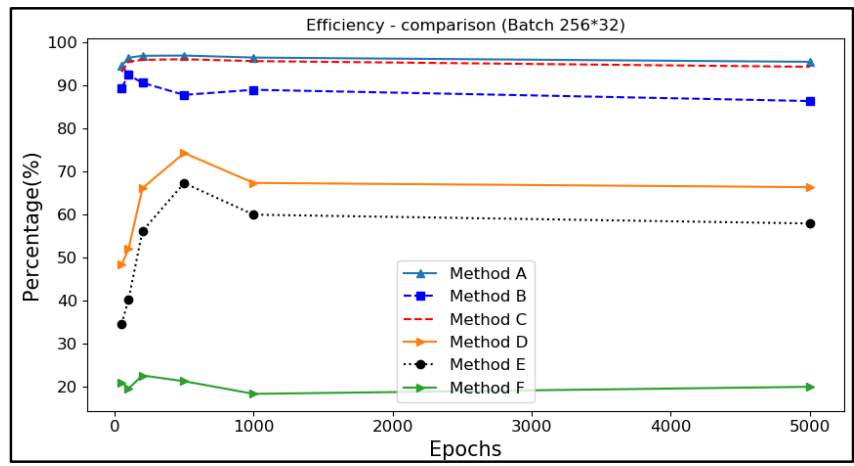

Fig. 12. Efficiency Percentage Comparison for the Batch $256 * 32$.

Both GPU4 and Standalone PC models in Method D are faster than the GPU1 and GPU8 models. When considering Method D in Batch $256 * 32$ and Batch $256 * 8$, the efficiency percentage of the GPU4 model in Batch 256*32 is higher than the Batch $256 * 8$. When consider Method E for Batch $256 * 32$ the Standalone PC model is noticeably faster than the GPU8 model hence, Method E ratio is the lowest ratio for the Batch $256 * 32$. The GPU8 model in Method F required more time for the training compared to the GPU4 model while results of the Method $\mathrm{F}$ in Batch $256 * 32$ is faster than Batch $256 * 8$. However, the ratio of Method $\mathrm{F}$ is higher than Method $\mathrm{E}$ for the Batch $256 * 32$.

\section{DISCUSSION}

This study aims to predict real-time Cryptocurrency Price by using Deep Learning algorithm whereas exploiting IoT concepts and beyond using Cloud computing and Data Parallelism. Time consumption is the major barrier for the 
training a large data set sequenced in the neural network. Therefore, this application primarily concerned to develop an algorithm to forecast the Cryptocurrencies price prediction accuracy. Numerous research experts discussed cloud computing [15, 17], Deep learning algorithms [18], cryptocurrency price prediction, Bitcoin $[6,7,9]$ and data parallelism [19, 20] separately as three different topics. Thus, it has a potential and significant correlation between these three approaches and can be experimented together to explain precise model for the Cryptocurrencies price prediction. However, this potential was ignorance and created a substantial gap in the field. Therefore, the experimental methodology of this study combined these three studies into a single platform to exploit the IoT basic concept for real-time Cryptocurrencies price prediction based on historical data. The main challenge of the real-time Cryptocurrencies price predicts models is that the application accuracy in real-world due to the fluctuating nature of the Cryptocurrencies. Similarly, identifying daily trends in the Bitcoin market while gaining insight into optimal features surrounding Bitcoin price is important because they try to predict the sign of the regular price change with the highest possible accuracy [9]. The Bayesian Neural Networks are a precise approach to estimate the maximum likelihood of Cryptocurrencies price and explaining the high volatility of the recent Bitcoin price [7]. Alternatively, reduce the training time of the Deep Learning algorithm is a noteworthy challenge for the cryptocurrency price prediction approaches. However, without Cryptocurrencies price prediction accuracy, computation time useless.

This study identified three major gaps in the cryptocurrencies price prediction models through the literature review as (1) accuracy of the application (2) long computation time and (3) application of IoT concepts to the prediction models. Concerning all the gaps in the current Cryptocurrencies price prediction applications, this study developed four hybrid architecture models, namely, (i) Standalone PC, (ii) GPU1 - without data parallelism model, (iii) GPU4 - with data parallelism model, and (iv) GPU8 - with data parallelism model for Cryptocurrencies training methods with a similar deep learning algorithm.

Primarily, the study concerned to enhance the accuracy of the Cryptocurrencies price prediction model and suggests an alternative to overcome the factors effect to reduce the prediction accuracy using Deep learning algorithm. The study utilized 266,776 historical data for the training of Cryptocurrencies price prediction Deep learning algorithm. The experiment has maintained maximum epoch for the Deep learning algorithm training as 5000 because the study expected to achieve more than $80 \%$ of price prediction accuracy. This study applied IoT technology combined with the Cloud computing to predict Cryptocurrencies price and to train the Cryptocurrencies price prediction, model. Also, the volume of the data set considerably influence to the accuracy and computation time of the prediction models and thus used five different batch sizes for the experiment. The accuracy percentage of the prediction and volume of the data set has a positive correlation which means the prediction of the big data set can be higher compared to the small volume of data set.
The Google ML engine provides different types of GPU for Cloud computing with data parallelism models which can be utilized for Deep Learning training. Therefore, types of GPU may have potential to reduce the computation time and accuracy of the training methods. The main advantage of the parallelism data is that it can be divided into a few batches to reduce the data set size of one batch, and then GPU can compute an individual quantity of the data set. However, the reduction of the volume of the data set that simulated to GPU affected to the prediction accuracy. Furthermore, the study identified that accuracy of the Cryptocurrency price prediction models can be increased using fully connected dense neural network with ReLU activation function in hidden layers and linear activation function in the output layer.

Deep Learning algorithm training is a highly timeconsuming process when the data set is large. Subsequently, this study combined the IoT concept with parallel processing and Deep Learning to reduce the computation time of the prediction of the models by training the historical data over pre-determined time slots. Firstly, the Standalone PC model was trained, and highest prediction accuracy which $88.7 \%$ was obtained by Batch 32 within $765.69 \mathrm{~min}$. The best accuracy percentage for this model was $81.27 \%$ and this could be achieved within $59.78 \mathrm{~min}$. by the Batch $256^{*} 16$. Secondly, the GPU1 - without data parallelism model was trained. The Batch 32 reported the highest prediction accuracy as $88.7 \%$ but it consumed 1785.97 min which is not practical. However, Batch $256^{*} 16$ has the best efficiency which is $99.84 \mathrm{~min}$ and accuracy percentage was 81.27 for this model. Thirdly, the GPU4 - with data parallelism model trained and Batch $256^{* 4}$ represented $87.78 \%$ of accuracy within $909.85 \mathrm{~min}$. For this model Batch, 256*32 has the best efficiency which is 22.58 min and accuracy was $79.09 \%$. Finally, the GPU8 - with data parallelism model trained and $87.07 \%$ the highest accuracy percentage could be observed from Batch 32 within $686.54 \mathrm{~min}$. Batch $256 * 32$ has the best efficiency which is $28.23 \mathrm{~min}$ for $79.09 \%$ of accuracy. All four models achieved almost $80 \%$ Cryptocurrencies price prediction accuracy.

The experimental results confirmed that the GPU4 - with data parallelism and the GPU8 - with data parallelism models can reduce the computation time which is approximately within 30 minutes for the large batch sizes. Few authors applied the Deep Learning approach with the parallel neural network [17], data parallelism [15] and Parallel Consensual Neural Networks [20] to reduce the computation time. Similarly, [19] has discussed the effect of traffic flow in cloud computing for the computation time using different types of parallel architectures. All these studies proved that a combination of Cloud computing with data parallelism for the training model significantly reduce the computation time. However, the data parallelism models can be executed for a large set of historical data, and Deep Learning training with the different GPU types available on the Google ML engine. Furthermore, proposed hybrid architecture models can be utilized in any IoT application. Correspondingly, future experiments can be focused on device parallelism with cloud computing (GPU-8) for the Deep Learning training. Besides, understanding decentralized approaches for big data databases $[21,22]$, decision making utilizing predicting techniques [23, 
24], could be an inspiring method to make the Internet of Things into one of the future Fourth Industrial Revolution Technologies (4IR/FIR).

\section{CONCLUSION}

This study trained four hybrid architecture models to predict real-time Cryptocurrencies price using deep learning algorithm by exploiting the IoT concept. The experimental results confirmed that Cloud computing technology stimulus to secure new trends by performing parallel computing in IoT. Similarly, the results of this study confirmed that data parallelism and Deep Learning algorithm-based Cryptocurrencies price prediction models can reduce computation time up to $90 \%$ with $80 \%$ of accuracy. However, the comparison between the model which did not train with data parallelism namely the Standalone PC and the GPU1 without data parallelism models revealed the insignificant outcome. The Batch $256 * 32$ in GPU8 - without data has the best accuracy which is $79.09 \%$. The GPU4 - without data parallelism model resulted in similar results and the Batch $256 * 32$ reported $79.09 \%$ of accuracy. These values revealed that the potential of Cloud computing with data parallelism (GPU-8 and GPU-4) models to use for Cryptocurrencies price prediction. Therefore, the experimental results concluded that uses of Cloud computing with data parallelism (GPU-4 and GPU-8) models can accelerate the Cryptocurrencies price prediction process than all other hybrid architecture models tested in this study and this may vary with the size of the batch. Ultimately, there is an enormous potential to apply the proposed hybrid architecture models into any other deep learning models such as character recognition, the biomedical field, in addition to any application in IoT such as industrial automation and natural disaster prediction.

\section{AUTHOR'S CONTRIBUTION}

A.P. and M.N.H. conceived the study idea and developed the analysis plan. A.P. analyzed the data and wrote the initial paper. M.N.H. helped to prepare the figures and tables and finalizing the manuscript. R.S. completed the final editing of the manuscript. All authors read the manuscript.

\section{REFERENCES}

[1] A. Rosic, (2016). What is Cryptocurrency: Everything You Need to Know [Ultimate Guide]. Retrieved from Blockgeeks.com: https://blockgeeks.com/guides/what-is-cryptocurrency.

[2] H. Yan, P. Yu, D. Long (2019). Study on Deep Unsupervised Learning Optimization Algorithm Based on Cloud Computing. In 2019 International Conference on Intelligent Transportation, Big Data \& Smart City, pp 679-681.

[3] Cloud ML Engine Overview. (2018). Retrieved from Google.com: https://Cloud computing.google.com/ml-engine/docs/tensorflow/ techni cal-overview

[4] L. Song, J. Mao, Y. Zhuo, X. Qian, H. Li, Y. Chen (2019). HyPar: Towards Hybrid Parallelism for Deep Learning Accelerator Array. In 2019 IEEE International Symposium on High Performance Computer Architecture, pp. 56-68.

[5] [G. Onoufriou, R. Bickerton, S. Pearson, G. Leontidis (2019). Nemesyst: A Hybrid Parallelism Deep Learning-Based Framework Applied for Internet of Things Enabled Food Retailing Refrigeration Systems. arXiv preprint arXiv:1906.01600.
[6] D. Sheehan, (2017). Predicting Cryptocurrency Prices with Deep Learning. Retrieved from Github: https://dashee87.github.io/deep\% 20learning/python/predicting-cryptocurrency-prices-with-deep-learning/

[7] H. Jang, J. Lee, "An Empirical Study on Modelling and Prediction of Bitcoin Prices with Bayesian Neural Networks based on Blockchain Information," IEEE Early Access Articles, vol. 99, pp. 1-1, 2017.

[8] S. McNally, "Predicting the price of Bitcoin using machine learning," School Comput., Nat. College Ireland, Dublin, Ph.D. dissertation 2016.

[9] S. Velankar, S. Valecha, S. Maji. (2018). Bitcoin Price Prediction using Machine Learning. International Conference on Advanced Communications Technology, 144-147, 11-14 Feb. 2018.

[10] I. Georgoula, D. Pournarakis, C. Bilanakos, D. Sotiropoulos, G. M. Giaglis (2015). Using time-series and sentiment analysis to detect the determinants of bitcoin prices, SSRN Electronic Journal.

[11] C. G. AkcoraAsim, K. Dey, A. Dey, Y. R. Gel, M. Kantarcioglu (2018) Forecasting Bitcoin Price with Graph Chainlets, Advances in Knowledge Discovery and Data Mining.

[12] Matta, M., Lunesu, I., \& Marchesi, M. (2015). Bitcoin Spread Prediction Using Social and Web Search Media. In UMAP Workshops.

[13] D. Steinkrau, P. Y. Simard, and I. Buck (2005) Using GPUs for machine learning algorithms in Proceedings of the Eighth International Conference on Document Analysis and Recognition. IEEE Computer Society, pp. 1115-1119.

[14] Cireşan, D. C., Meier, U., Gambardella, L. M., \& Schmidhuber, J. (2010). Deep, big, simple neural nets for handwritten digit recognition. Neural computation, 22(12), 3207-3220.

[15] A. Krizhevsky, (2014). One weird trick for parallelizing convolutional neural networks. arXiv:1404.5997, 1-7.

[16] M. Whitney, (2016). Deep Learning with Multiple GPUs on Rescale: Torch. Retrieved from Blog.rescale.com: https://blog.rescale.com/deeplearning-with-multiple-gpus-on-rescale-torch/

[17] F. Åström, R. Koker. (2011). A parallel neural network approach to prediction of Parkinson's Disease. Expert Systems with Applications, 12470-12474.

[18] A. A. Diro and N. Chilamkurti, "Distributed attack detection scheme using deep learning approach for Internet of Things", Future Generation Computer Systems, Volume 82, May 2018, Pages 761-768.

[19] P. Sekwatlakwatla, M. Mphahlele, T. Zuva. (2016). Traffic Flow Prediction in Cloud Computing. International Conference on Advances in Computing and Communication Engineering, 123-128, 28-29 Nov. 2016.

[20] J. Ekanayake, X. Qiu, T. Gunarathne, Scott Beason, Geoffrey Fox. (n.d.). High Performance Parallel Computing with Cloud and Cloud. 139.

[21] S. Kalid, A. Syed, A. Mohammad, and M. N. Halgamuge, "Big-Data NoSQL Databases: Comparison and Analysis of "Big-Table", "DynamoDB", and "Cassandra", IEEE 2nd International Conference on Big Data Analysis, Beijing, China, pp 89-93, 10-12 March 2017.

[22] V. Vargas, A. Syed, A. Mohammad, and M. N. Halgamuge, "Pentaho and Jaspersoft: A Comparative Study of Business Intelligence Open Source Tools Processing Big Data to Evaluate Performances", International Journal of Advanced Computer Science and Applications (IJACSA), Volume 7, Issue 10, pp 20-29, November 2016.

[23] A. A. R. Madushanki, M. N. Halgamuge, W. A. H. S. Wirasagoda, and A. Syed, "Adoption of the Internet of Things (IoT) in Agriculture and Smart Farming towards Urban Greening: A Survey", International Journal of Advanced Computer Science and Applications (IJACSA), Volume 10, No. 4, pp 11-28, April 2019.

[24] A. Singh, M. N. Halgamuge, R. Lakshmiganthan, "Impact of Different Data Types on Classifier Performance of Random Forest, Naïve Bayes, and k-Nearest Neighbors Algorithms", International Journal of Advanced Computer Science and Applications (IJACSA), Volume 8, No 12, pp 1-10, December 2017. 